\title{
Metastatic Disease, Myeloma, and Lymphoma Affecting the Hip
}

\author{
Arianna L. Gianakos, DO ${ }^{1}$ \\ Jay N. Patel, DO ${ }^{2}$ \\ James C. Wittig, MD2 \\ ${ }^{1}$ Department of Orthopaedic Surgery, Jersey City Medical Center - \\ RWJBarnabas Health, Jersey City, New Jersey \\ ${ }^{2}$ Division of Orthopaedic Oncology, Department of Orthopaedic \\ Surgery, Morristown Medical Center - Atlantic Health System, \\ Morristown, New Jersey \\ ${ }^{3}$ Department of Orthopaedic Surgery, Hackensack University Medical \\ Center, Hackensack, New Jersey \\ J Hip Surg 2019;3:34-40.
}

Justin M. Miller, DO ${ }^{1}$

Laura Sonnylal, BS 3

\begin{abstract}
Address for correspondence Jay N. Patel, DO, Division of Orthopaedic Oncology, Department of Orthopaedic Surgery, Morristown Medical Center - Atlantic Health System, Carol G. Simon Cancer Center $2^{\text {nd }}$ Floor, 100 Madison Avenue, Morristown, NJ 07960 (e-mail: jaypatel37@gmail.com).
\end{abstract}
Abstract
Keywords
- orthopaedic oncology
- malignant
- metastatic disease
- multiple myeloma
- lymphoma

The three most common causes for bony lesions in patients over 40 years of age are metastatic disease, multiple myeloma, and lymphoma. Oftentimes, these conditions have a predilection for the hip region. The authors present the incidence, characteristics, and treatment of metastatic disease, multiple myeloma, and lymphoma affecting the hip joint to help clinicians accurately diagnose and manage these conditions. They also present the senior author's technique of cemented long stem hemiarthroplasty for treating these conditions when they affect the proximal femur.
Orthopaedic surgeons are responsible for knowing how to properly manage patients with a suspected underlying malignancy and its sequela when it affects the bones, particularly in the hip joint. This can include knowing when to refer to an orthopaedic oncologist to perform biopsies, stabilize impending fractures, and fix pathologic fractures as a result of these types of tumors. Although pain in the hip can result from numerous pathologies, suspicious lesions from metastatic disease, myeloma, or lymphoma render proper evaluation and workup critical. We present the incidence, characteristics and treatment of metastatic disease, multiple myeloma (MM), and lymphoma affecting the hip joint to help clinicians accurately diagnose and manage these conditions.

\section{Multiple Myeloma}

Multiple myeloma is the most common primary bone cancer, primarily affecting patients over the age of 40 years. MM occurs due to an abnormal proliferation of plasma cells within the bone marrow, an accumulation that results in an increase in osteoclast activity along with a decrease in osteoblast activity. When there is a solitary plasma cell tumor either within the bone or in the soft tissues, it is known as a plasmacytoma. While the exact etiology of MM is unknown, there have been several cytogenetic mutations linked to this condition, with mutation of chromosome $14 q 32$, hyperdiploidy, and deletion of chromosome 13 being the three most common mutations. ${ }^{1,2}$ Sergentanis et al performed a systematic review of meta-analyses demonstrating strong associations with occupational risk factors including firefighters, hairdressers, and farmers; as well as, pesticide exposure, obesity, pernicious anemia, and ankylosing spondylitis. ${ }^{3}$

The most common symptom in patients with MM is bone pain, occurring in more than two-thirds of patients at the time of presentation. ${ }^{4}$ The increase in osteoclast activity leads to osteolytic lesions, which severely weaken the bone and can lead to fractures. In a study by Melton et al, $60 \%$ of patients with MM suffered a pathologic fracture with $40 \%$ of patients developing such a fracture within the first year after diagnosis. $^{5}$ They noted an association between oral received

August 31, 2018

accepted after revision

November 6, 2018

published online

February 15, 2019
Copyright $\odot 2019$ by Thieme Medical

Publishers, Inc., 333 Seventh Avenue, New York, NY 10001, USA

Tel: +1(212) 584-4662.
DOI https://doi.org/ 10.1055/s-0039-1678743. ISSN 2472-8446. 
corticosteroid use, elevated serum calcium levels, and chemotherapy use with the development of fractures.

The diagnosis of MM is based on both laboratory and imaging studies. Patients with MM have several laboratory abnormalities, including the appearance of immunoglobulin light chains (subunit of antibodies), also known as Bence Jones proteins, in their urine. On blood testing, they may have hypercalcemia, leukopenia, anemia, and thrombocytopenia. The diagnosis of MM is confirmed with a bone marrow biopsy that reveals an abnormal number of plasma cells.

Up to $90 \%$ of MM patients have osteolytic bone lesions. ${ }^{6}$ The pelvis is affected in up to $34 \%$ of patients. ${ }^{7}$ Radiographs of various bones in the body are taken to search for additional bone lesions. Upon radiographic examination, these osteolytic lesions have the appearance of being "punched-out" of the bone with the absence of reactive sclerosis. Several studies have shown that low dose computed tomography (CT) scans are superior to plain radiographs in detecting osteolytic bone lesions in MM..$^{8-10}$ Magnetic resonance imaging (MRI), positron emission tomography, or CT can be used to identify unsuspected bone lesions, differentiate pathologic fractures from osteoporotic fractures, and identify extramedullary disease. ${ }^{11-14}$

While there are currently no curative treatments for MM, advances have been made to help increase survival and decrease the disease burden. According to the International Myeloma Working Group, all patients should be treated with bisphosphonate therapy. ${ }^{15,16}$ Intravenous bisphosphonates are administered every 3 to 4 weeks to help prevent pathologic fractures and other skeletal-related events. In a recent network meta-analysis by Mhaskar et al, the authors found that bisphosphonates were associated with lower rates of pathological fractures and skeletal-related events compared with a placebo or no treatment in patients with MM. ${ }^{17}$ For MM patients with renal dysfunction, denosumab can be used as an alternative. ${ }^{18}$ Plasmacytomas can be treated with radiation alone. Other indications for radiation include pathologic fractures, tumors causing compression of neurovascular structures, and areas of bone pain that are unresponsive to other forms of treatment. The use of stem cell transplantation, chemotherapy, immunomodulatory agents, and other drugs has also been reviewed in the literature. $^{19-21}$

Orthopaedic surgical intervention is reserved for patients with pathologic fractures, impending fractures of the long bones, vertebral column instability, as well as for the secondary effects of osteolytic lesions such as joint degeneration (when life expectancy is $>3$ months). ${ }^{16,22}$ Surgical intervention is generally well tolerated and may improve survival time. ${ }^{23}$ There are several classification systems for lesions around the hip joint. ${ }^{24-26}$ Lesions in the pelvis and periacetabular region can present with large cavitary defects. Pain and difficulty with ambulation are present when the weight-bearing portion of the acetabulum is affected. Depending on the size of the lesion and the extent of bone loss, patients may require total hip arthroplasty, a jumbo cup, the use of augments, or the placement of a cage. Adjuvant therapy consisting of highly concentrated phenol, electrocauterization, argon, methyl-methacrylate, liquid nitrogen, and radiation can also be used to help improve sterilization of the margins. Patients with a plasmacytoma, a negative bone marrow biopsy, and those with no paraproteinemia in their serum or lower staging have a better prognosis after surgery. ${ }^{27}$ Younger patients have a more favorable survival rate. ${ }^{28}$ Patients undergoing large reconstructions around the hip joint have a higher rate of dislocation as well as neurovascular injury. ${ }^{29}$

\section{Lymphoma}

Lymphoma is a malignant neoplasia of lymphocytes with various subtypes, the non-Hodgkin type being the most common in the bone. Primary bone lymphoma accounts for $5 \%$ of all non-Hodgkin lymphomas ${ }^{30}$ and $2.6 \%$ of all primary bone tumors. ${ }^{31}$ Cases of primary bone Hodgkin's lymphoma and secondary Hodgkin's lymphoma in its later stages involving the bone have also been reported in the literature. $^{32-34}$ The more common secondary lymphoma of bone occurs when there is skeletal involvement of the metastatic disease. Skeletal involvement occurs in 16 to $20 \%$ of lymphoma patients. ${ }^{35}$

While the exact cause of lymphoma remains unclear, several subtypes have been linked to viral and bacterial infections, chemical exposures, and certain diseases. ${ }^{36-42}$ There have been various staging systems proposed, with the Ann Arbor system being one of the most commonly used. ${ }^{43}$ This staging system is based on the location of the malignancy and the presence of systemic symptoms. The presence of systemic symptoms is given a letter B designation in the staging system. B Symptoms, as they are called, include weight loss $>10 \%$ of normal body weight, night sweats, and fevers. As more information is gathered from ongoing research, the Ann Arbor Staging System has been modified to include the presence of bulky disease and regions of lymph node involvement. ${ }^{44}$

Lymphoma presents as an osteolytic lesion on plain radiographs. There may be subtle permeative destruction of the bone, which can be easily missed. As lymphoma usually involves the soft tissue as well, MRI can be used to better visualize and define the soft tissue components (-Fig. 1). Diagnosis is confirmed with a biopsy and subsequent flow cytometry analysis of the soft tissue component. A multidrug chemotherapy regimen is the mainstay in the treatment of lymphoma. Current chemotherapy regimens include cyclophosphamide, doxorubicin, vincristine, and prednisone (CHOP) or CHOP plus rituximab (RCHOP). Radiation therapy may be used in select situations, including when a compromising level of bone destruction is present. Surgery is generally reserved for pathologic fractures, lesions compromising the integrity of a joint, and avascular necrosis. In a series of primary bone lymphoma surgeries performed by Demircay et al, the only prognostic factor found to be significantly associated with survivorship was age. ${ }^{45}$ Beal et al found that patients treated with a combination of chemotherapy (CHOP or RCHOP) and radiation had increased survival rates and superior outcomes. ${ }^{46}$ 


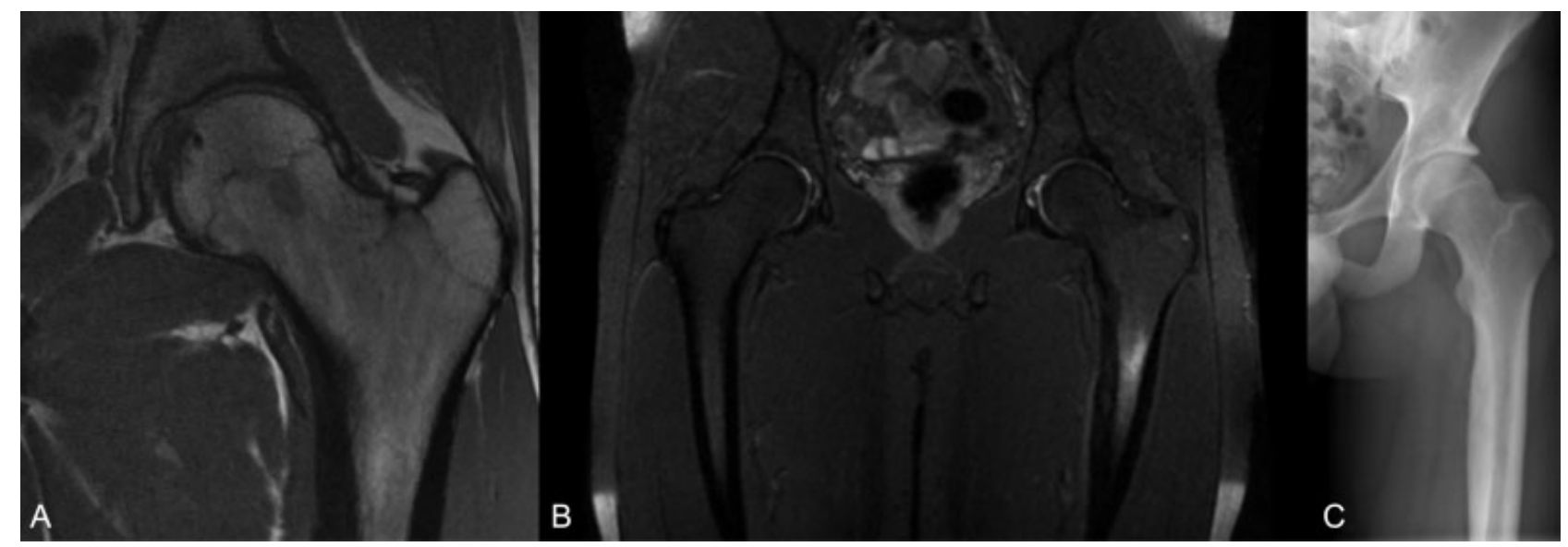

Fig. $1 \mathrm{~T} 1$ (A) and T2 (B) weighted magnetic resonance imaging and plain radiograph (C) demonstrating diffuse large B cell lymphoma affecting the proximal femur.

\section{Metastatic Disease}

Metastatic cancer is generally found in patients over the age of 40 years, with bones being the third most common sites for cancer metastasis. The most common cancers that metastasize to bone are breast, lung, thyroid, renal, and prostate cancer. ${ }^{47}$ The thoracic spine is the most common site of bony metastasis followed by the proximal femur. The proximal femur is the most common site of fracture due to metastatic bone lesions. About half of the pathologic fractures in the proximal femur are located in the femoral neck, 30\% are located in the subtrochanteric region, and $20 \%$ are located in the intertrochanteric region. ${ }^{48}$ Depending on the type of primary cancer, the metastatic disease can cause osteolytic lesions, osteosclerotic lesions or mixed osteolytic-osteosclerotic lesions. Cancers that cause osteolytic lesions include breast, lung thyroid, renal, and MM. Osteosclerotic lesions are due to increased osteoblastic activity as seen in metastatic breast and prostate cancers. Mixed metastatic lesions can be seen with lymphoma but also with breast and lung cancers. In a study performed by Gainor and Buchert, the overall fracture healing rate for patients with metastatic bone disease was 35\%. ${ }^{49}$ In patients surviving longer than 6 months, their fracture union rate was $74 \%$; the use of internal fixation improved the rate of fracture healing by $23 \%$ compared with cast immobilization alone. They concluded that a life expectancy of longer than 6 months was the primary factor determining fracture healing in patients.

Imaging should begin with plain radiographs of the entire pelvis and femur. Judet views and inlet/outlet views of the pelvis should also be obtained to help assess the extent of the lesion around the pelvis and acetabulum. A CT scan of the pelvis and femur can help provide better visualization of the lesion and the amount of bone destruction. A CT scan should be performed with thin slices to help better visualize the characteristics of the lesion. An MRI can provide details of the lesion as well as any soft tissue components (-Fig. 2). Furthermore, an MRI can help evaluate the location of nearby neurovascular structures to help guide surgical decisionmaking.

Diagnosis of metastatic disease is confirmed with biopsy. All patients without a history of cancer or metastatic disease should undergo a biopsy to identify the tumor and formulate a subsequent treatment plan. Patients with a remote history of cancer or those with no history of metastatic disease should also undergo a biopsy to determine whether there is the presence of metastatic disease from the known cancer, a new primary tumor, or metastatic disease from a new tumor.

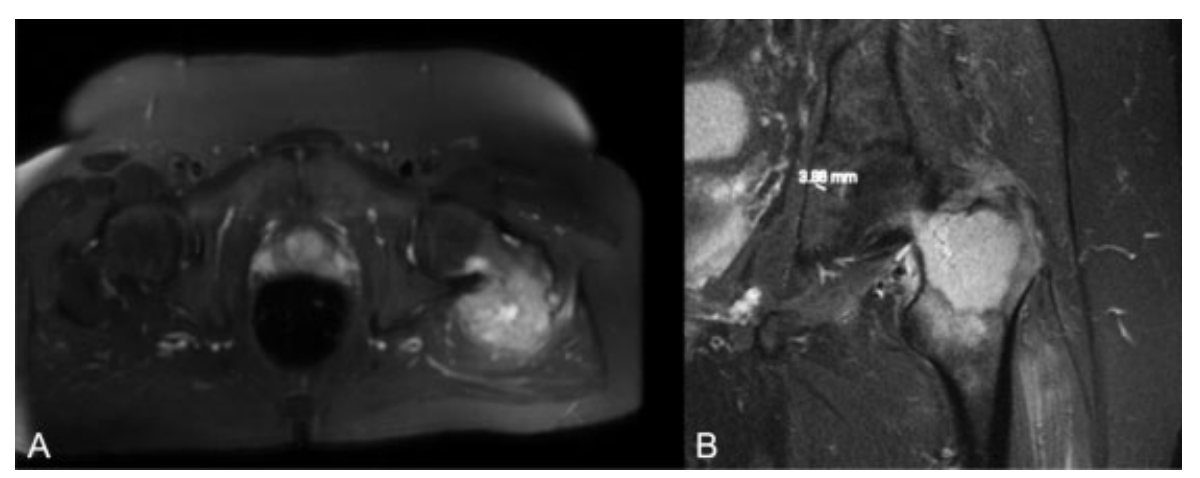

Fig. 2 T1-weighted axial (A) and coronal (B) magnetic resonance imaging demonstrating metastatic renal cell (clear cell type with sarcomatoid features) affecting the intertrochanteric region of the left proximal femur. On the axial cut (A), there is a soft tissue component to the lesion posteriorly. 
Patients with a known history of recent, active metastatic disease do not need a biopsy.

Symptomology is similar to the other tumors described in this article. With regard to the hip, osteolytic lesions in the proximal femur and acetabulum can lead to severe pain with ambulation and an eventual pathologic fracture. Patients with incurable disease, who are unsafe surgical candidates due to medical deterioration, may be treated with radiation. Patients with an impending fracture or pathologic fracture are treated with intramedullary fixation or joint reconstruction (-Fig. 3). Those with a Mirels' criteria score $\geq 8$ can be treated with prophylactic fixation. ${ }^{50}$ Patients with metastatic disease can also be treated with bisphosphonates to help reduce bone pain, pathologic fractures, and other skeletal-related events. ${ }^{51,52}$ Monoclonal human antibodies such as denosumab have also been found to be efficacious in reducing skeletal-related events compared with bisphosphonate therapy alone. ${ }^{52-55}$ Because metastases from the kidneys and thyroid are very vascular, preoperative embolization of bone lesions should be considered to help decrease intraoperative bleeding. Studies have shown that 70 to $100 \%$ of the tumor needs to be devascularized during the embolization process to achieve the best results in terms of decreased intraoperative blood loss in patients with bony renal cell carcinoma metastases. ${ }^{56,57}$

\section{Surgical Treatment-Review of the Literature}

The incidence of bony metastasis in the advanced stages of certain malignancies has been reported to range from 25 to $100 \%{ }^{58}$ Pathologic fractures have been reported in up to 8 to $30 \%$ of patients with bony metastases with higher risks when the proximal femur is involved. ${ }^{59}$ In their series of
142 patients with a pathologic femur fracture, Sarahrudi et al reported the 1-year survival after surgical treatment to be $17 \%{ }^{60}$ The aim of treatment for metastatic lesions of the bone is to provide a stable construct that allows for immediate weight bearing and prevents progression of the fracture. For patients with pathologic fractures as a result of metastatic lesions, the goal is to provide secure fixation that restores function and that will outlast the patient. The method of surgical treatment depends on the location of the metastatic lesion. For periarticular disease involving the proximal femur, recent literature has demonstrated a preference for endoprosthetic reconstruction when compared with reconstruction nails or other osteosynthetic devices.

Endoprosthetic reconstruction can improve patient ambulatory status, decrease pain, and afford the surgeon the ability to removed gross disease, minimize recurrence, and protect the femur against progressing disease. Peterson et al evaluated the outcomes after utilization of a long stem hemiarthroplasty in the setting of bony metastasis of the proximal femur and found fair levels of postoperative function. ${ }^{61}$ Steensma et al reported a significantly lower treatment failure rate and fewer revisions requiring implant exchange in patients who underwent endoprosthetic reconstruction when compared with open reduction-internal fixation and intramedullary nailing. ${ }^{62}$ Janssen et al performed a retrospective study on the complications after the surgical management of proximal femoral metastasis. ${ }^{63}$ They compared intramedullary nailing, open reduction and internal fixation, and endoprosthetic reconstruction and discovered that revision rates and overall systemic complications did not differ between the three treatment strategies. Failure of fixation was most common after open reduction

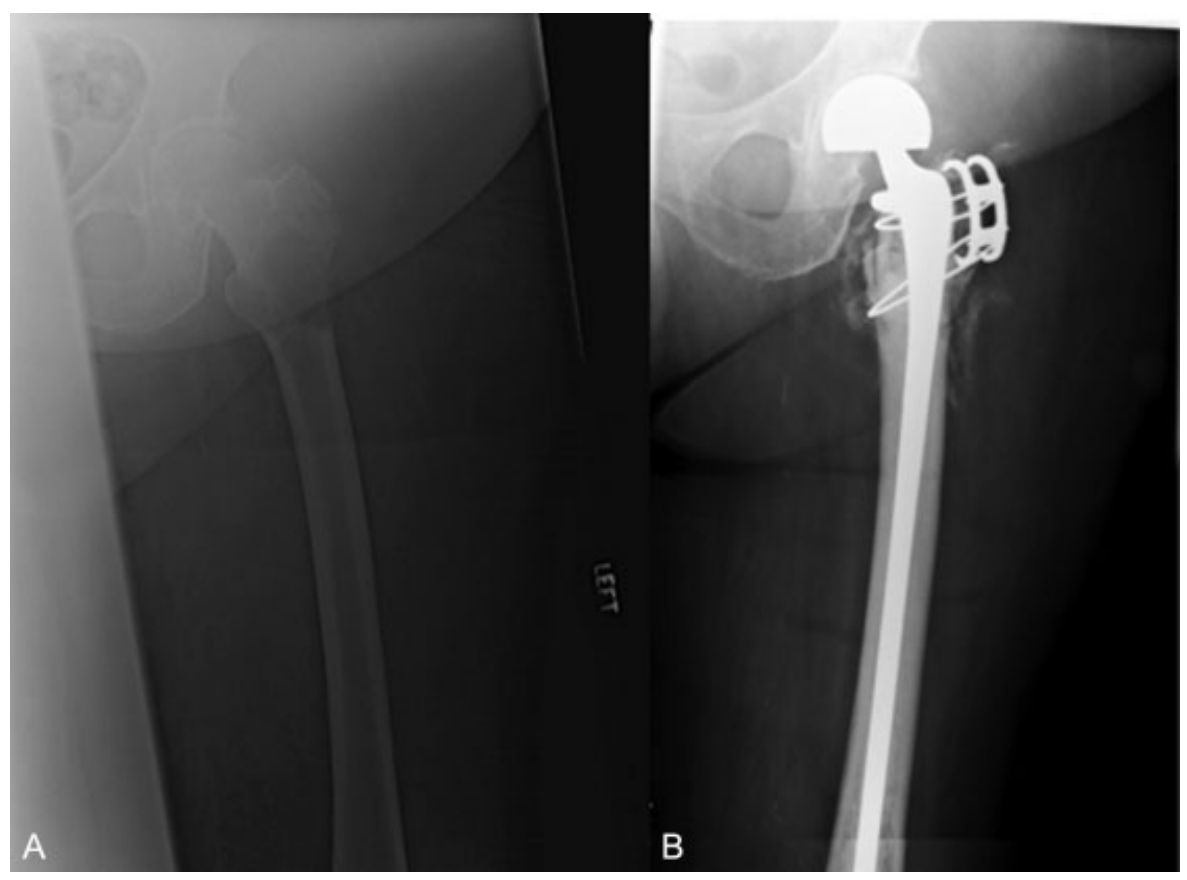

Fig. 3 (A) Plain radiograph demonstrating an osteolytic lesion in the trochanteric region of the left proximal femur from metastatic disease. (B) Postoperative radiograph of left femur demonstrating a cemented long stem hemiarthroplasty with a short claw plate with cerclage wires to secure the greater trochanter to preserve the attachment of the hip abductors. 
with internal fixation. Lastly, reports have demonstrated good outcomes and a reduction in failure rates with the use of methyl methacrylate as a supplement in proximal femur fixation. ${ }^{64}$

Classically, the treatment of metastatic carcinoma, lymphoma, or myeloma involves protecting the entire bone using either an intramedullary nail or arthroplasty for pathologic fracture or impending fracture of the proximal femur. Recent studies suggest similar postoperative outcomes when addressing the isolated segment of bone with a reduction in adverse consequences including hypotension, oxygen desaturation, embolization, coma, and even death. However, Alvi and Damron demonstrated higher failure rates with instrumentation of a lesser anatomic extent of the bone and therefore continue to support use of protection of the entire bone when treating impending or pathologic fractures of the femur. 22

\section{Cemented Long Stem Hip Hemiarthroplasty Technique}

It has been the senior author's (J.C.W.) preference to utilize a long stem cemented hemiarthroplasty whenever feasible for impending or actual pathological fractures of the proximal femur. The procedure enables the surgeon to remove all gross disease, minimize recurrence and hardware failure, and protect almost the entire femur.

A standard posterolateral approach to the hip is preferred. The piriformis muscle as well as the short external rotators is released from their insertion on the greater trochanter and tagged with a strong nonabsorbable suture. These are then retracted posteriorly which protects the sciatic nerve and exposes the hip capsule. A T-shaped capsulotomy is preformed, with a tag suture also placed in each leaf. The hip is then dislocated, exposing the femoral neck for the subsequent cut. This is performed with a sagittal saw from the piriformis fossa to $\sim 1$ fingerbreadth proximal to the lesser trochanter. Gross tumor tissue is debrided using rongeurs and curettes. Additionally, attention is directed to the intramedullary endosteal surface of the proximal femur, which is also intensely debrided with a curette to further remove diseased tissue. A pulsatile canal irrigator and brush are then utilized inside the medullary canal. Next, the canal is reamed after placing a ball tipped guidewire to the distal aspect of the femur. To properly ream for the cement mantle, the canal is reamed to $2 \mathrm{~mm}$ greater than the size of the stem in $1 \mathrm{~mm}$ increments. The ball-tipped guidewire is used to measure the length of the stem and the proximal femur is then broached in the standard fashion. The corresponding trial stem is placed to ensure proper fit and is then removed. The canal is cleansed once more with pulsed lavage and hydrogen peroxide soaked vaginal packing is placed in the canal to remove any remaining blood products. The cement is then mixed using three bags of polymethylmethacrylate and allowed to harden to a dough-like consistency. The canal is then injected retrograde from distal to proximal with the cement in a nonpressurized manner. The final prosthesis (-Fig. 4) is inserted slowly while maintaining proper

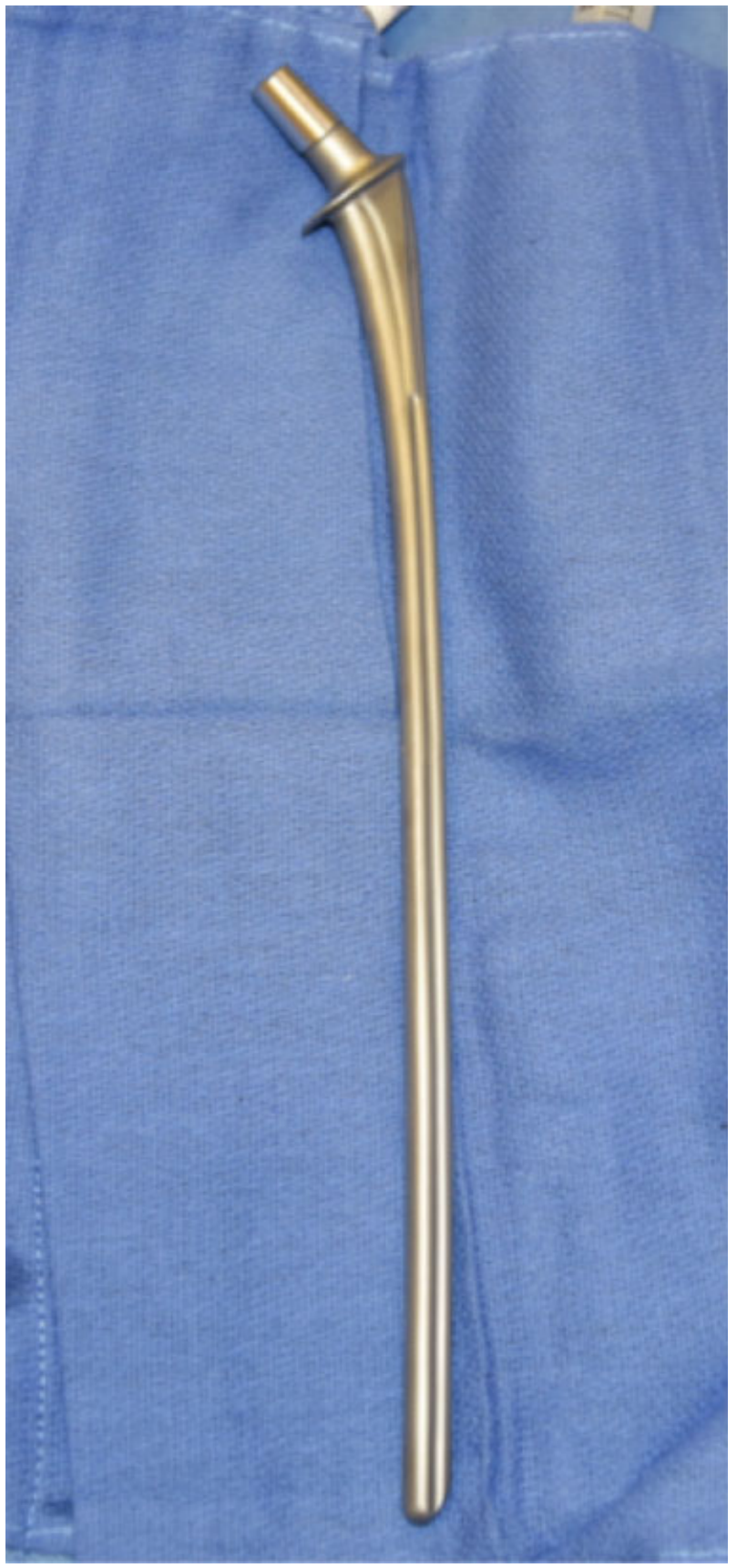

Fig. 4 Photograph of a long-stemmed femoral implant for a hemiarthroplasty.

femoral anteversion and is held in place until the cement hardens. Not pressurizing the cement in this manner is believed to decrease venous extravasation from the femoral canal, thus reducing the risk of cardiopulmonary collapse. Next, a trial femoral head is placed, and the hip is reduced to ensure proper fit. The hip is taken through a full range of motion and deemed to be stable without dislocation. The hip is then dislocated, the trial component removed, and the final bipolar femoral head prosthesis is malleted into place to engage the Morse taper. Lastly, the hip is reduced once more, taken through another range of motion, and the tagged hip capsule is closed tightly with the piriformis and short external rotators (-Fig. 5). The surgical site is then closed 


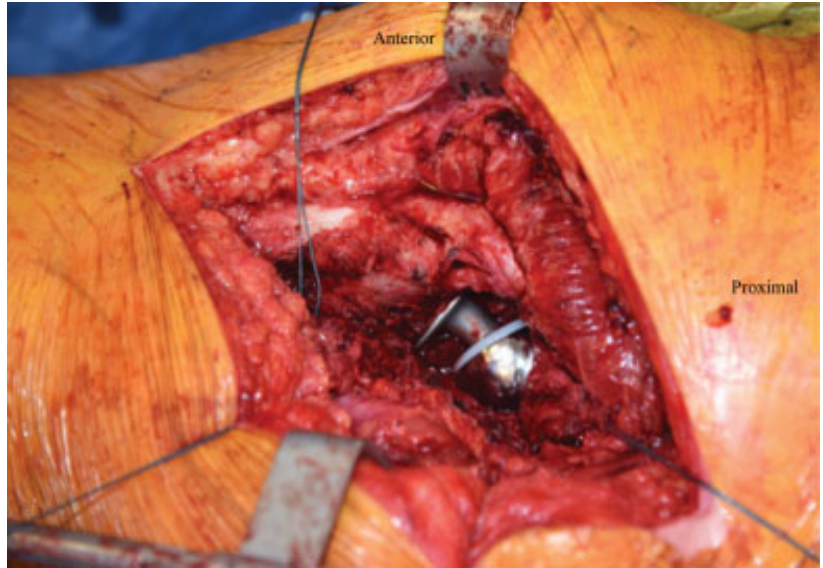

Fig. 5 Photograph showing the final implant after it has been reduced into the acetabulum. Note the sutures used to tag the capsule and short external rotator muscles during the approach.

in a layered fashion and the patient is placed in an abduction pillow with posterior hip precautions.

\section{Conclusion}

Although treatment of malignant bone tumors of the proximal femur resulting from metastasis, myeloma, or lymphoma remains a challenge, endoprosthetic replacement of the proximal femur provides a treatment strategy with good functional and oncological outcomes. The use of a cemented hip hemiarthroplasty, intramedullary nail fixation, or open reduction-internal fixation procedures can help restore function and improve the overall quality of life.

\section{Conflict of Interest}

None.

\section{References}

1 Decaux O, Lodé L, Minvielle S, Avet-Loiseau H. [Genetic abnormalities in multiple myeloma: role in oncogenesis and impact on survival]. Rev Med Interne 2007;28(10):677-681

2 Avet-Loiseau $\mathrm{H}$. Role of genetics in prognostication in myeloma. Best Pract Res Clin Haematol 2007;20(04):625-635

3 Sergentanis TN, Zagouri F, Tsilimidos G, et al. Risk factors for multiple myeloma: a systematic review of meta-analyses. Clin Lymphoma Myeloma Leuk 2015;15(10):563-77.e1, 3

4 Rajkumar SV, Kyle RA. Treatment of multiple myeloma and related disorders. Cambridge; New York: Cambridge University Press; 2009

5 Melton LJ III, Kyle RA, Achenbach SJ, Oberg AL, Rajkumar SV. Fracture risk with multiple myeloma: a population-based study. J Bone Miner Res 2005;20(03):487-493

6 Roodman GD. Mechanisms of bone metastasis. N Engl J Med 2004; 350(16):1655-1664

7 Kyle RA. Multiple myeloma: review of 869 cases. Mayo Clin Proc 1975;50(01):29-40

8 Gleeson TG, Moriarty J, Shortt CP, et al. Accuracy of whole-body low-dose multidetector CT (WBLDCT) versus skeletal survey in the detection of myelomatous lesions, and correlation of disease distribution with whole-body MRI (WBMRI). Skeletal Radiol 2009;38(03):225-236

9 Princewill K, Kyere S, Awan O, Mulligan M. Multiple myeloma lesion detection with whole body CT versus radiographic skeletal survey. Cancer Invest 2013;31(03):206-211
10 Wolf MB, Murray F, Kilk K, et al. Sensitivity of whole-body CT and MRI versus projection radiography in the detection of osteolyses in patients with monoclonal plasma cell disease. Eur J Radiol 2014;83(07):1222-1230

11 Moulopoulos LA, Dimopoulos MA, Weber D, Fuller L, Libshitz HI, Alexanian R. Magnetic resonance imaging in the staging of solitary plasmacytoma of bone. J Clin Oncol 1993;11(07):1311-1315

12 Baur A, Stäbler A, Brüning R, et al. Diffusion-weighted MR imaging of bone marrow: differentiation of benign versus pathologic compression fractures. Radiology 1998;207(02):349-356

13 Zamagni E, Nanni C, Patriarca F, et al. A prospective comparison of $18 \mathrm{~F}$-fluorodeoxyglucose positron emission tomography-computed tomography, magnetic resonance imaging and wholebody planar radiographs in the assessment of bone disease in newly diagnosed multiple myeloma. Haematologica 2007;92 (01):50-55

14 Regelink JC, Minnema MC, Terpos E, et al. Comparison of modern and conventional imaging techniques in establishing multiple myeloma-related bone disease: a systematic review. Br J Haematol 2013;162(01):50-61

15 Terpos E, Morgan G, Dimopoulos MA, et al. International Myeloma Working Group recommendations for the treatment of multiple myeloma-related bone disease. J Clin Oncol 2013;31(18): 2347-2357

16 Palumbo A, Sezer O, Kyle R, et al; IMWG. International Myeloma Working Group guidelines for the management of multiple myeloma patients ineligible for standard high-dose chemotherapy with autologous stem cell transplantation. Leukemia 2009;23 (10):1716-1730

17 Mhaskar R, Kumar A, Miladinovic B, Djulbegovic B. Bisphosphonates in multiple myeloma: an updated network meta-analysis. Cochrane Database Syst Rev 2017;12:CD003188

18 Raje N, Terpos E, Willenbacher W, et al. Denosumab versus zoledronic acid in bone disease treatment of newly diagnosed multiple myeloma: an international, double-blind, doubledummy, randomised, controlled, phase 3 study. Lancet Oncol 2018;19(03):370-381

19 Scharschmidt TJ, Lindsey JD, Becker PS, Conrad EU. Multiple myeloma: diagnosis and orthopaedic implications. J Am Acad Orthop Surg 2011;19(07):410-419

20 Steinbach M, Richards T, Faiman B. Strategies for selecting the optimal treatment in newly diagnosed multiple myeloma patients. Semin Oncol Nurs 2017;33(03):254-264

21 Mateos MV, San Miguel JF. Management of multiple myeloma in the newly diagnosed patient. Hematology (Am Soc Hematol Educ Program) 2017;2017(01):498-507

22 Alvi HM, Damron TA. Prophylactic stabilization for bone metastases, myeloma, or lymphoma: do we need to protect the entire bone? Clin Orthop Relat Res 2013;471(03):706-714

23 Utzschneider S, Schmidt H, Weber P, Schmidt GP, Jansson V, Dürr HR. Surgical therapy of skeletal complications in multiple myeloma. Int Orthop 2011;35(08):1209-1213

24 Harrington KD. New trends in the management of lower extremity metastases. Clin Orthop Relat Res 1982;(169):53-61

25 Levy RN, Sherry HS, Siffert RS. Surgical management of metastatic disease of bone at the hip. Clin Orthop Relat Res 1982;(169): 62-69

26 D'Antonio JA, Capello WN, Borden LS, et al. Classification and management of acetabular abnormalities in total hip arthroplasty. Clin Orthop Relat Res 1989;(243):126-137

27 Angtuaco EJ, Fassas AB, Walker R, Sethi R, Barlogie B. Multiple myeloma: clinical review and diagnostic imaging. Radiology 2004;231(01):11-23

28 Ludwig H, Durie BG, Bolejack V, et al. Myeloma in patients younger than age 50 years presents with more favorable features and shows better survival: an analysis of 10549 patients from the International Myeloma Working Group. Blood 2008;111(08): 4039-4047 
29 Parikh SN, Kreder HJ. Pelvic reconstruction for massive acetabular insufficiency. Clin Orthop Relat Res 2005;(434):217-221

30 Freeman C, Berg JW, Cutler SJ. Occurrence and prognosis of extranodal lymphomas. Cancer 1972;29(01):252-260

31 Limb D, Dreghorn C, Murphy JK, Mannion R. Primary lymphoma of bone. Int Orthop 1994;18(03):180-183

32 Newcomer LN, Silverstein MB, Cadman EC, Farber LR, Bertino JR, Prosnitz LR. Bone involvement in Hodgkin's disease. Cancer 1982; 49(02):338-342

33 Binesh F, Mirjalili MR, Akhavan A, Navabii H. Primary bony Hodgkin's lymphoma. BMJ Case Rep 2012;2012:201

34 Tang F, Min L, Ye Y, et al. Classic Hodgkin lymphoma in pelvis: a case report highlights diagnosis and treatment challenges. Medicine (Baltimore) 2017;96(39):e8196

35 Fletcher CDM, World Health Organization. International Agency for Research on Cancer. WHO Classification of Tumours of Soft Tissue and Bone. 4th ed. Lyon: IARC Press; 2013

36 Auerbach A, Aguilera NS. Epstein-Barr virus (EBV)-associated lymphoid lesions of the head and neck. Semin Diagn Pathol 2015;32(01):12-22

37 Vockerodt M, Yap LF, Shannon-Lowe C, et al. The Epstein-Barr virus and the pathogenesis of lymphoma. J Pathol 2015;235(02): 312-322

38 Linke-Serinsöz E, Fend F, Quintanilla-Martinez L. Human immunodeficiency virus (HIV) and Epstein-Barr virus (EBV) related lymphomas, pathology view point. Semin Diagn Pathol 2017;34 (04):352-363

39 Paydas S. Hepatitis C virus and lymphoma. Crit Rev Oncol Hematol 2015;93(03):246-256

40 Schinasi L, Leon ME. Non-Hodgkin lymphoma and occupational exposure to agricultural pesticide chemical groups and active ingredients: a systematic review and meta-analysis. Int J Environ Res Public Health 2014;11(04):4449-4527

41 Czarnota J, Gennings C, Colt JS, et al. Analysis of environmental chemical mixtures and non-Hodgkin lymphoma risk in the $\mathrm{NCI}-$ SEER NHL study. Environ Health Perspect 2015;123(10):965-970

42 Lauby-Secretan B, Loomis D, Grosse Y, et al; WHO International Agency for Research on Cancer. Carcinogenicity of polychlorinated biphenyls and polybrominated biphenyls. Lancet Oncol 2013;14(04):287-288

43 Carbone PP, Kaplan HS, Musshoff K, Smithers DW, Tubiana M. Report of the committee on Hodgkin's disease staging classification. Cancer Res 1971;31(11):1860-1861

44 Lister TA, Crowther D, Sutcliffe SB, et al. Report of a committee convened to discuss the evaluation and staging of patients with Hodgkin's disease: Cotswolds meeting. J Clin Oncol 1989;7(11): 1630-1636

45 Demircay E, Hornicek FJ Jr, Mankin HJ, Degroot H III. Malignant lymphoma of bone: a review of 119 patients. Clin Orthop Relat Res 2013;471(08):2684-2690

46 Beal K, Allen L, Yahalom J. Primary bone lymphoma: treatment results and prognostic factors with long-term follow-up of 82 patients. Cancer 2006;106(12):2652-2656

47 Habermann ET, Sachs R, Stern RE, Hirsh DM, Anderson WJ Jr. The pathology and treatment of metastatic disease of the femur. Clin Orthop Relat Res 1982;(169):70-82
$48 \mathrm{Sim}$ FH. Metastatic bone disease of the pelvis and femur. Instr Course Lect 1992;41:317-327

49 Gainor BJ, Buchert P. Fracture healing in metastatic bone disease. Clin Orthop Relat Res 1983;(178):297-302

50 Mirels H. Metastatic disease in long bones. A proposed scoring system for diagnosing impending pathologic fractures. Clin Orthop Relat Res 1989;(249):256-264

51 Hultborn R, Gundersen S, Ryden S, et al. Efficacy of pamidronate in breast cancer with bone metastases: a randomized, double-blind placebo-controlled multicenter study. Anticancer Res 1999;19 (4C):3383-3392

52 O'Carrigan B, Wong MH, Willson ML, Stockler MR, Pavlakis N, Goodwin A. Bisphosphonates and other bone agents for breast cancer. Cochrane Database Syst Rev 2017;10:CD003474

53 Gnant M, Mlineritsch B, Schippinger W, et al; ABCSG-12 Trial Investigators. Endocrine therapy plus zoledronic acid in premenopausal breast cancer. N Engl J Med 2009;360(07):679-691

54 Fizazi K, Lipton A, Mariette X, et al. Randomized phase II trial of denosumab in patients with bone metastases from prostate cancer, breast cancer, or other neoplasms after intravenous bisphosphonates. J Clin Oncol 2009;27(10):1564-1571

55 Stopeck AT, Lipton A, Body JJ, et al. Denosumab compared with zoledronic acid for the treatment of bone metastases in patients with advanced breast cancer: a randomized, double-blind study. J Clin Oncol 2010;28(35):5132-5139

56 Sun S, Lang EV. Bone metastases from renal cell carcinoma: preoperative embolization. J Vasc Interv Radiol 1998;9(02): 263-269

57 Chatziioannou AN, Johnson ME, Pneumaticos SG, Lawrence DD Carrasco $\mathrm{CH}$. Preoperative embolization of bone metastases from renal cell carcinoma. Eur Radiol 2000;10(04):593-596

58 Coleman RE. Skeletal complications of malignancy. Cancer 1997; 80(8, Suppl):1588-1594

59 Menendez L OKU Musculoskeletal Tumors(Orthopaedic Knowledge Update). 1st ed. Rosemont, IL: American Academy of Orthopaedic Surgeons; 2001

60 Sarahrudi K, Greitbauer M, Platzer P, Hausmann JT, Heinz T, Vécsei V. Surgical treatment of metastatic fractures of the femur: a retrospective analysis of 142 patients. J Trauma 2009;66(04): 1158-1163

61 Peterson JR, Decilveo AP, O'Connor IT, Golub I, Wittig JC. What are the functional results and complications with long stem hemiarthroplasty in patients with metastases to the proximal femur? Clin Orthop Relat Res 2017;475(03):745-756

62 Steensma M, Boland PJ, Morris CD, Athanasian E, Healey JH. Endoprosthetic treatment is more durable for pathologic proximal femur fractures. Clin Orthop Relat Res 2012;470(03): 920-926

63 Janssen SJ, Kortlever JT, Ready JE, et al. Complications after surgical management of proximal femoral metastasis: a retrospective study of 417 patients. J Am Acad Orthop Surg 2016;24 (07):483-494

64 Harrington KD, Sim FH, Enis JE, Johnston JO, Diok HM, Gristina AG. Methylmethacrylate as an adjunct in internal fixation of pathological fractures. Experience with three hundred and seventy-five cases. J Bone Joint Surg Am 1976;58(08):1047-1055 